\section{MYCORRHIZAL SYMBIOSIS}

\section{Setting the mood}

Nat. Commun. 11, 2114 (2020).

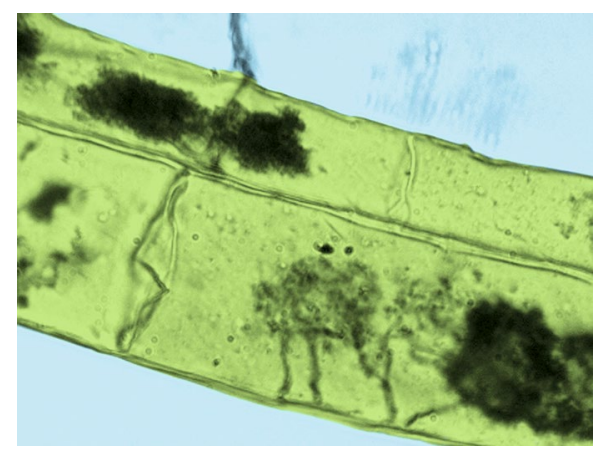

Credit: Science History Images / Alamy Stock Photo

Most plants recruit the assistance of fungi to help them take up essential nutrients, such as phosphorus, from the soil. When such nutrients are scarce, plants and fungi can form extremely intimate associations called arbuscular mycorrhizal symbioses, but this is not without cost to the plant in the form of considerable amounts of carbon compounds. Jeongmin Choi of the Department of Plant Sciences, University of Cambridge, UK, and colleagues have now discovered that a protein called SUPPRESSOR OF MAX2-1 (SMAX1) acts as a molecular brake to control plants' willingness to engage with fungi.

At the beginning of any relationship, the prospective partners start by engaging in a conversation to 'check out' each other's suitability and willingness to 'hook up'. For plants and fungi, these 'chat-up lines' take the form of an exchange of chemical signals. The researchers found that rice plants defective in SMAX1 were more highly colonized following inoculation with the fungus Rhizophagus irregularis than wild-type individuals. Without functional SMAX1, signals produced by the fungus set off a series of changes in the rice that prepare the plant for the physical advances of its suitor and also cause the synthesis and release of strigolactone, which acts as a signal to the fungus to progress the interaction.

SMAX1 does not directly detect the chemical messages sent by the fungus. This is done by an intermediary receptor called Dwarf14Like in rice, whose homologue in other plants, including Arabidopsis, is known to recognize karrikins - complex organic molecules found in the smoke from wildfires. This work places SMAX1 at a junction between karrikin and strigolactone signalling, and at a node through which nutritional status can adjust a plant's desire for interspecies relationships.

\section{Chris Surridge}

Published online: 5 May 2020 https://doi.org/10.1038/s41477-020-0676-5 